\title{
BIS/MC ALGORITHM BASED 3D-QSAR FOR RECEPTOR MODELLING TO EXPLORE ANTI-MALARIAL ACTIVITY OF SYNTHETIC
} PRODIGININES

\author{
V. H. Masanda*, D. T. Mahajana, S. A. Quazia, T. B. Haddab, \\ a Department of Chemistry, Vidya Bharati College, Camp, Amravati, \\ Maharashtra, India. \\ b Laboratoire Chimie des Matériaux, Université Mohammed Premier, \\ Oujda-60000, Morocco. \\ For all correspondence; e-mail: vijaymasand@gmail.com and \\ vijaymasand@rediffmail.com Tel: 0091-9403312628
}

\begin{abstract}
:
3D-QSAR analysis using BiS/MC (Biological Substrate/Multi-Conformational) algorithm has been performed in the present work to explore anti-malarial activity of synthetic prodiginines along with the modelling of the characteristics features of the receptor site. Using the novel approach implemented in the $\mathrm{BiS} / \mathrm{MC}$, it has been observed that lipophilic and electrostatic are prominent interactions between the prodiginines and the receptor site. BiS/MC builds statistically sound 3D-QSAR model to construct pseudoreceptor site. The analysis of the generalized self-consistent complementary field constructed within BiS/MC approach reveals introduction of $\mathrm{H}$-bonding capable groups on side chain and lipophilic groups on pyrrole ring B could result in enhancement of anti-malarial activity of prodiginines.
\end{abstract}

Keywords: Prodiginines, Anti-malarial activity, 3D-QSAR, BiS/MC algorithm

Abbreviations: BiS/MC- Biological Substrate/Multi-Conformational

\section{Introduction:}

Malaria, a global health challenge especially for tropical and subtropical countries, kills more than 2 million peoples every year (http://www.who.int/malaria/en/) [1]. The emergence of resistance against existing drugs is worsening the situation. To curb malaria, ongoing modification of existing drugs and search for new therapeutics has lead to identification of novel chemical compounds viz. xanthones, 
artimisins, prodiginines etc., a few to mention [2-8], as hopeful antimalarial agents.

Prodiginines (figure 1), the red pigment tripyrrole derivatives [9], have received considerable attention of researchers due to in vitro moderate to high activity against Plasmodium species at a very low concentration, oral administration, marked parasite clearance and cures in some cases without evident weight loss but search for synthetic analogues with better anti-malarial activity and with reduced toxicity persists [9-11]. To achieve these objectives, modern techniques like QSAR, GUSAR, molecular docking, pharmacophore modelling etc. are promising tools [28].

Fig. 1. Synthetic prodiginines analysed in present study

The mechanism of action against $P$. falciparum and the receptor with which the prodiginines interact is unknown [9-11] therefore 3D-QSAR could be especially trustworthy. In present work, we have carried out 3DQSAR analysis using BiS/MC algorithm. BiS/MC, a novel approach for 3D-QSAR, is a superimposing algorithm which allows one to construct a pseudo-atomic model of a receptor and to calculate the interaction energy of each conformer with the obtained model receptor [12-17], whereas the traditional 3D-QSAR approaches like CoMFA, CoMSIA, HASL etc. are able to give idea about the common pattern of fields around the congener series of molecules only. Thus BiS/MC has 
additional advantages apropos of receptor site over conventional 3DQSAR approaches.

\section{Results and Discussions}

\subsection{BiS/MC analysis of anti-malarial activity of Prodiginines:}

The statistical characteristics for BiS/MC 3D-QSAR analysis for 53 synthetic prodiginines are as follows: $R=0.972, R^{2}=0.945, \operatorname{cross} R^{2}=$ 0.884 and $S=0.431$. The analysis of the statistical characteristics shows that the model developed by $\mathrm{BiS} / \mathrm{MC}$ is statistically reliable and can be used as a novel tool for future drug design. A straight line relation between the actual and predicted $\mathrm{pIC}_{50}$ indicates that the $\mathrm{BiS} / \mathrm{MC}$ model has a good predictive efficiency.

\subsection{Analysis of BiS/MC output:}

Figure 2 represents the output of $\mathrm{BiS} / \mathrm{MC}$ for anti-malarial activity of prodiginines. Though the BiS/MC analysis was carried for all 53 synthetic prodiginines, only four most active and four least active have been depicted as representatives in figure 3 for comparison purpose.

From figure 2, it is clear that the major factors which govern the interactions of prodiginines with receptor are lipophilic and electrostatic in nature. In the case of the most active compounds number 40 and $\mathbf{4 1}$, though the interactions are appearing identical, but the difference in their $\mathrm{IC}_{50}$ values (0.9 and $1.3 \mathrm{nM}$ respectively) reveals that some other factors like $\log P$, pKa etc. should be considered. Similar explanations can be applied for compound number $\mathbf{2 7}$ and $\mathbf{2 8}$ with identical $\mathrm{IC}_{50}(-0.23$ $\mathrm{nM}$ ), even though there is a considerable difference in their structure and it emerges out that they interact with same atoms of the receptor in an identical manner. 
Fig. 2. Output of BiS/MC

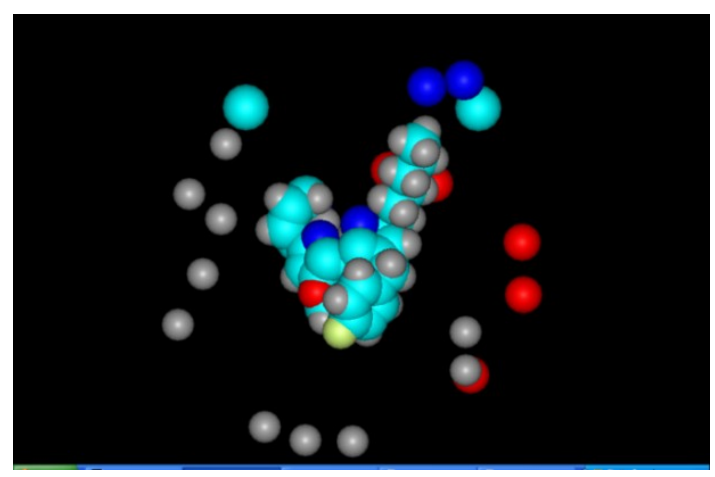

Molecule no. 40:

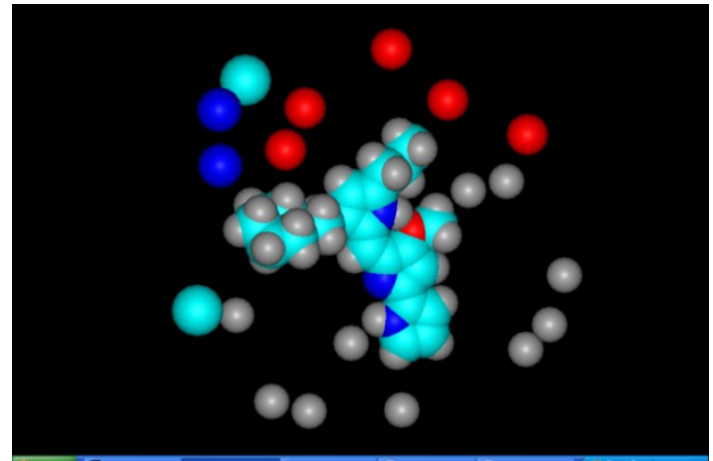

Molecule no. 27:

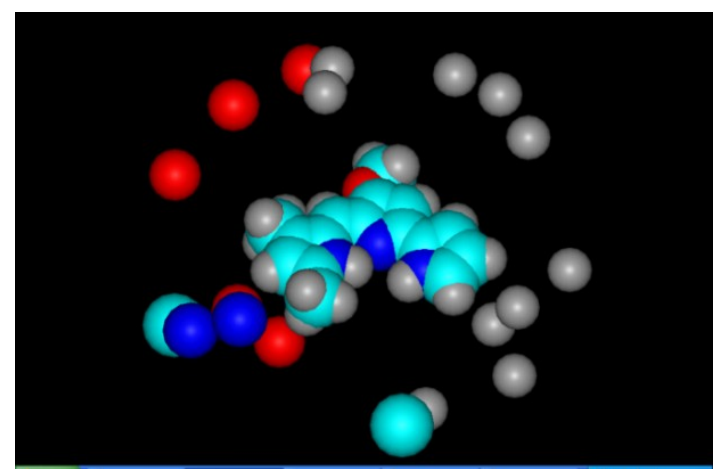

Molecule no. 24:

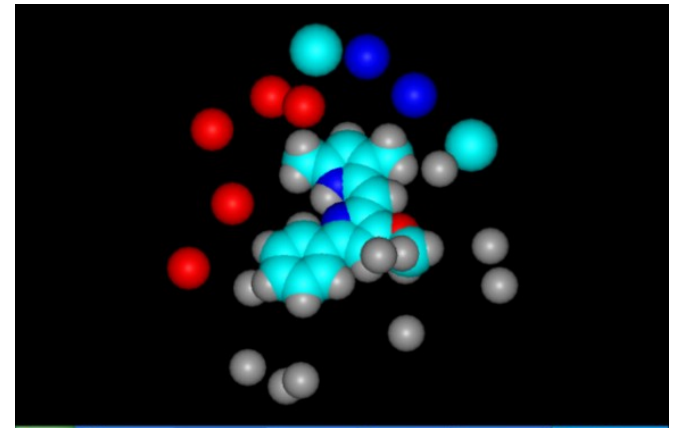

Molecule no. 4:

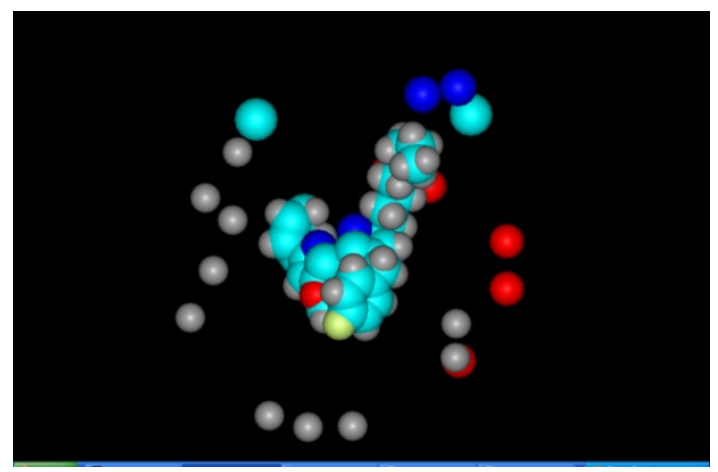

Molecule no.41:

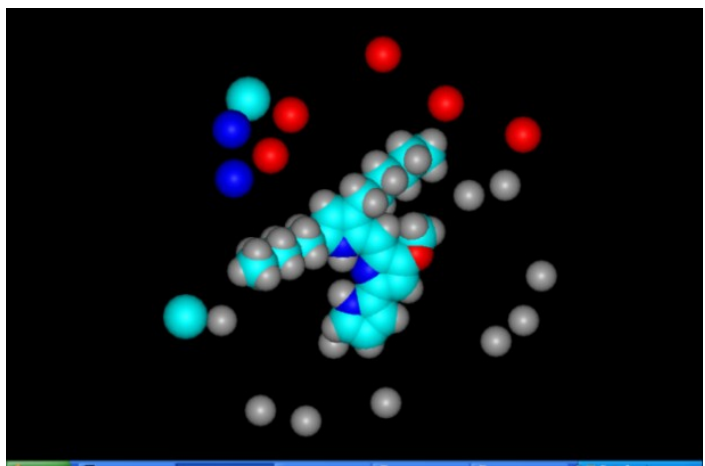

Molecule no. 28:

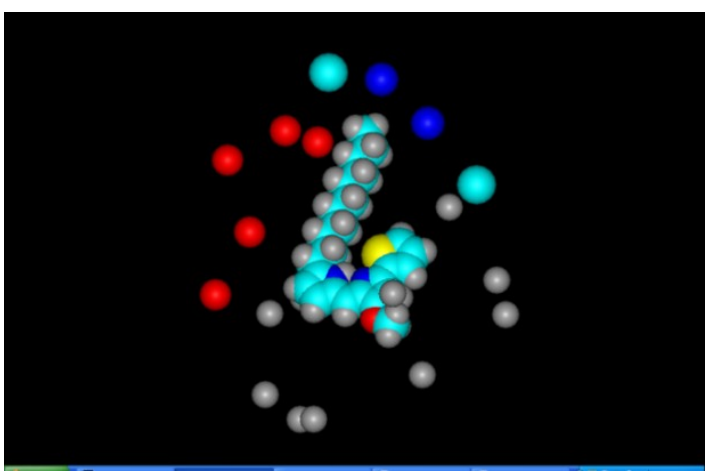

Molecule no. 6:

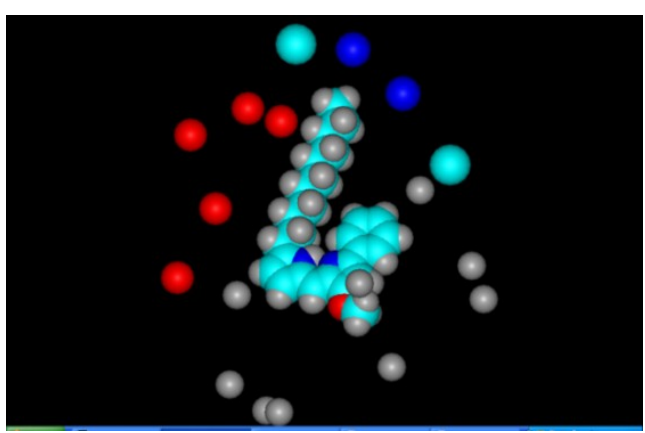

Molecuel no. 3: 
A possible explanation for different probe spheres is: Red is negatively charged pseudo-atom (oxygen-like); blue is also negatively charged pseudo-atom but with less charge and greater radius (nitrogen-like); gray is positively charged pseudo-atom (like a hydrogen of $-\mathrm{OH},-\mathrm{NH},-\mathrm{COOH}$ groups); cyan is non-charged "lipophilic" pseudoatom (like alkyl carbon or hydrogen) [12-17].

\section{Materials and methods:}

3.1 Data set: In present work, fifty three structurally diverse synthetic prodiginines possessing substituents like $-\mathrm{F},-\mathrm{Cl}$, alkyl, $-\mathrm{NH}_{2}$, etc. at different positions were selected from literature [9]. All were assayed for their in vitro anti-malarial activity against $P$. falciperum pansensitive D6 with chloroquine (CQ) as a reference drug. To obtain a symmetrically distributed data for smoother $\mathrm{BiS} / \mathrm{MC}$ analysis, the data reported as $\mathrm{IC}_{50}$ was converted to $-\log \mathrm{IC}_{50}\left(\mathrm{pIC}_{50}\right)[10]$. A complete list of compounds is presented in table 1 along with experimental $\mathrm{IC}_{50}(\mathrm{nM})$. The experimental $\mathrm{pIC}_{50}$ along with predicted $\mathrm{pIC}_{50}$ by BiS/MC are listed in table 1.

Table 1 Experimental data and predicted $\mathrm{IC}_{50}$ by BiS/MC

\begin{tabular}{|c|c|c|c|c|c|}
\hline $\begin{array}{ll}\text { Molecule } & R_{1} \\
\text { no. }\end{array}$ & $\overline{\mathbf{R}_{2}}$ & $\mathbf{R}_{\mathbf{3}}$ & $\begin{array}{l}\mathrm{IC}_{50}(\mathrm{nM}) \\
\mathrm{D} 6\end{array}$ & $\begin{array}{l}\text { Actual } \\
\text { pIC }\end{array}$ & $\begin{array}{l}\text { Predicted } \\
\text { pIC } 50\end{array}$ \\
\hline 1 & $\overline{\mathrm{CH}_{3}}$ & $\mathrm{CH}_{3}$ & 4250 & -3.628 & -3.65013 \\
\hline 2 & $\mathrm{n}-\mathrm{C}_{11} \mathrm{H}_{23}$ & $\mathrm{H}$ & 4060 & -3.608 & -3.76422 \\
\hline 3 & $\mathrm{n}-\mathrm{C}_{11} \mathrm{H}_{23}$ & $\mathrm{H}$ & 10470 & -4.019 & -3.67714 \\
\hline 4 & $\mathrm{CH}_{3}$ & $\mathrm{CH}_{3}$ & 19410 & -4.288 & -3.92714 \\
\hline 5 & $\mathrm{n}-\mathrm{C}_{11} \mathrm{H}_{23}$ & $\mathrm{H}$ & 2920 & -3.465 & -3.17274 \\
\hline 6 & $\mathrm{CH}_{3}$ & $\mathrm{CH}_{3}$ & $>25000$ & -3.773 & -3.47111 \\
\hline 7 & n- $\mathrm{C}_{11} \mathrm{H}_{23}$ & $\mathrm{H}$ & 5940 & -3.361 & -3.32064 \\
\hline 8 & $\mathrm{CH}_{3}$ & $\mathrm{CH}_{3}$ & $>25000$ & -3.25 & -3.58345 \\
\hline
\end{tabular}




\begin{tabular}{|c|c|c|c|c|c|}
\hline 9 & $\mathrm{n}-\mathrm{C}_{3} \mathrm{H}_{7}$ & $\mathrm{H}$ & 2300 & -2.574 & -2.06819 \\
\hline 10 & $\mathrm{n}-\mathrm{C}_{4} \mathrm{H}_{9}$ & $\mathrm{H}$ & 1780 & -1.903 & -2.17415 \\
\hline 11 & $\mathrm{n}-\mathrm{C}_{6} \mathrm{H}_{13}$ & $\mathrm{H}$ & 375 & -2.477 & -2.85857 \\
\hline 12 & $\mathrm{n}-\mathrm{C}_{8} \mathrm{H}_{17}$ & $\mathrm{H}$ & 80 & -3.23 & -3.39469 \\
\hline 13 & $\mathrm{n}-\mathrm{C}_{16} \mathrm{H}_{33}$ & $\mathrm{H}$ & 300 & -3.653 & -3.5456 \\
\hline 14 & $\mathrm{n}-\mathrm{C}_{11} \mathrm{H}_{22} \mathrm{NH}_{2}$ & $\mathrm{H}$ & 1700 & -2.662 & -2.40882 \\
\hline 15 & $\mathrm{H}$ & $\left(\mathrm{CH}_{2}\right)_{3} \mathrm{COOCH}_{3}$ & 4500 & -1.903 & -2.23137 \\
\hline 16 & $\mathrm{H}$ & $\mathrm{CH}_{2} \mathrm{CH}\left(\mathrm{CH}_{3}\right)_{2}$ & 460 & -1.447 & -1.16215 \\
\hline 17 & $\mathrm{H}$ & $\mathrm{n}-\mathrm{C}_{4} \mathrm{H}_{9}$ & 80 & -0.662 & -0.9224 \\
\hline 18 & $\mathrm{H}$ & $\mathrm{n}-\mathrm{C}_{6} \mathrm{H}_{13}$ & 28 & -0.903 & -1.01716 \\
\hline 19 & $\mathrm{H}$ & $\mathrm{n}-\mathrm{C}_{8} \mathrm{H}_{17}$ & 4.6 & -1.919 & -1.84772 \\
\hline 20 & $\mathrm{H}$ & $\mathrm{n}-\mathrm{C}_{10} \mathrm{H}_{21}$ & 8.0 & -2.23 & -2.35412 \\
\hline 21 & $\mathrm{H}$ & $\mathrm{n}-\mathrm{C}_{16} \mathrm{H}_{33}$ & $>25000$ & -1.812 & -1.62018 \\
\hline 22 & $\mathrm{H}$ & $\mathrm{C}_{6} \mathrm{H}_{5} \mathrm{CH}_{2}$ & 83 & -1.954 & -2.2573 \\
\hline 23 & $\mathrm{H}$ & $\begin{array}{l}\text { 4- } \\
\mathrm{OCH}_{3} \mathrm{C}_{6} \mathrm{H}_{4} \mathrm{CH}_{2}\end{array}$ & 170 & -1.748 & -1.72893 \\
\hline
\end{tabular}


26

27

28

29

30

31

32

33

34

35

36

37

38
$\mathrm{H}$

$\mathrm{CH}_{3}$

$\mathrm{n}-\mathrm{C}_{6} \mathrm{H}_{13}$

$\mathrm{n}-\mathrm{C}_{3} \mathrm{H}_{7}$

n- $\mathrm{C}_{8} \mathrm{H}_{17}$

$\mathrm{n}-\mathrm{C}_{3} \mathrm{H}_{7}$

$\mathrm{n}-\mathrm{C}_{3} \mathrm{H}_{7}$

n- $\mathrm{C}_{6} \mathrm{H}_{13}$

$n-\mathrm{C}_{6} \mathrm{H}_{13}$

n- $\mathrm{C}_{7} \mathrm{H}_{15}$

$n-\mathrm{C}_{6} \mathrm{H}_{13}$

n- $\mathrm{C}_{6} \mathrm{H}_{13}$

n- $\mathrm{C}_{8} \mathrm{H}_{17}$

n- $\mathrm{C}_{7} \mathrm{H}_{15}$

n- $\mathrm{C}_{8} \mathrm{H}_{17}$

n- $\mathrm{C}_{8} \mathrm{H}_{17}$

n- $\mathrm{C}_{8} \mathrm{H}_{17}$

92

$-0.477$

$-0.90233$
$-0.301$

$-0.40445$

$\mathrm{C}_{2} \mathrm{H}_{5}$

$\mathrm{n}-\mathrm{C}_{3} \mathrm{H}_{7}$
4- $\mathrm{ClC}_{6} \mathrm{H}_{4} \mathrm{CH}_{2}$

6.3

$-0.447$

$-0.452$

4- $\mathrm{ClC}_{6} \mathrm{H}_{4} \mathrm{CH}_{2}$
3 
40

$\mathrm{n}-\mathrm{C}_{7} \mathrm{H}_{15}$

4- $\mathrm{ClC}_{6} \mathrm{H}_{4} \mathrm{CH}_{2}$

2.8

0.045

0.03333

41

$\mathrm{n}-\mathrm{C}_{8} \mathrm{H}_{17}$

4- $\mathrm{ClC}_{6} \mathrm{H}_{4} \mathrm{CH}_{2}$

16

$-0.113$

$-0.36213$

42

4- $\mathrm{ClC}_{6} \mathrm{H}_{4} \mathrm{CH}_{2}$

3.9

$-0.462$

$-0.5408$

43

$\mathrm{n}-\mathrm{C}_{6} \mathrm{H}_{13}$

4- $\mathrm{FC}_{6} \mathrm{H}_{4} \mathrm{CH}_{2}$

0.9

$-0.602$

$-0.95763$

44

n- $\mathrm{C}_{8} \mathrm{H}_{17}$

4- $\mathrm{FC}_{6} \mathrm{H}_{4} \mathrm{CH}_{2}$

1.3

$-0.785$

$-0.95104$

45

n- $\mathrm{C}_{6} \mathrm{H}_{13}$

4- $\mathrm{BrC}_{6} \mathrm{H}_{4} \mathrm{CH}_{2}$

2.9

$-0.748$

$-0.71502$

46

n- $\mathrm{C}_{8} \mathrm{H}_{17}$

4- $\mathrm{BrC}_{6} \mathrm{H}_{4} \mathrm{CH}_{2}$

4

$-1.146$

$-1.15627$

47

48

49

50

51

52

53

2,4

2,4-

6.1

$-0.785$

$-0.87856$

4- $\mathrm{FC}_{6} \mathrm{H}_{4} \mathrm{CH}_{2}$

4- $\mathrm{FC}_{6} \mathrm{H}_{4} \mathrm{CH}_{2}$

5.6

$-0.919$

$-0.78514$

4- $\mathrm{BrC}_{6} \mathrm{H}_{4} \mathrm{CH}_{2}$

4- $\mathrm{BrC}_{6} \mathrm{H}_{4} \mathrm{CH}_{2}$

14

$-0.755$

$-0.93336$

4- $\mathrm{FC}_{6} \mathrm{H}_{4} \mathrm{CH}_{2}$

4- $\mathrm{ClC}_{6} \mathrm{H}_{4} \mathrm{CH}_{2}$

6.1

$-1.1$

$-0.72488$

4- $\mathrm{BrC}_{6} \mathrm{H}_{4} \mathrm{CH}_{2} \quad 4-\mathrm{ClC}_{6} \mathrm{H}_{4} \mathrm{CH}_{2}$

8.3

$-1.167$

$-0.66199$

$\mathrm{Cl}_{2} \mathrm{C}_{6} \mathrm{H}_{3} \mathrm{CH}_{2}$

$\mathrm{Cl}_{2} \mathrm{C}_{6} \mathrm{H}_{3} \mathrm{CH}_{2}$

12.6

$-0.556$

$-0.8125$ 
3.2 BiS/MC computations: The structures were drawn using ACD ChemSketch 12 freeware followed by optimization. The structures along with their experimental $\mathrm{pIC}_{50}$ data were used in BiS/MC. The standard procedure provided with BiS/MC was used to get the best results.

\section{Conclusions:}

The results of the analysis shows the following: (1) BiS/MC is very successful in building statistically robust 3D-QSAR models. (2) BiS/MC provides idea about the characteristics of receptor site. (3) Introduction of $\mathrm{H}$-bonding capable groups on side chain and lipophilic groups on pyrrole ring $B$ could result in enhancement of anti-malarial activity of prodiginines. (4) Lipophilic and electrostatic are prominent interactions between prodiginines and receptor site. The results of present analysis could be fruitful for future modifications in prodiginines to augment their anti-malarial activity.

\section{Acknowledgement}

Sincere thanks to Dr. F.C. Raghuwanshi, Principal, Vidya Bharati College, Amravati for providing other necessary laboratory facilities.

6. Conflict of interest: Authors declare no conflict of interests.

\section{References:}

http://www.who.int/malaria/en/

R.D. Jawarkar, V.H. Masand, K.N. Patil, D.T. Mahajan, M.H. Youssoufi, T.B. Hadda, and S.L. Kumbhare, (2010). 3D-QSAR Study on Coumarin Analogues as Potent Inhibitors of MAO-B using a COMFA Approach, Der Pharma Chemica. 2(6) 302-310.

D.T. Mahajan, R.D. Jawarkar, K.N. Patil, V.H. Masand, and G.M. Nazerruddin, (2010). 3D-QSAR studies on xanthone derivatives to understand pharmacological activities as MAO inhibitors, Der Pharma Chemica 2(4) 298-308.

V.H. Masand, R.D. Jawarkar, K.N. Patil, D.T. Mahajan, T.B. Hadda, and G.H. Kurhade, (2010). COMFA Analysis and toxicity risk assessment of coumarin analogues as MAO-A Inhibitors: Attempting better insight in drug design, Der Pharm Lettre 2(6) 350-357. 
V.H. Masand, R.D. Jawarkar, D.T. Mahajan, T.B. Hadda, A.M. Manikrao, P.N. Khatale, and J.V. Vyas, (2011). Presuming the Probable Antiinflammatory Mechanism of Ursolic Acid: a plant derived pentacyclic triterpenoid, using molecular docking, J. Comput. Method. Mol. Design. 1(2) 9-13.

V.H. Masand, R.D. Jawarkar, K.N. Patil, G.M. Nazerruddin, and S.O. Bajaj, (2010). Correlation potential of Wiener index vis-à-vis molecular refractivity: Antimalarial activity of xanthone derivatives, Org. Chem. An Ind. J. 6(1) 30-38.

V.H. Masand, R.D. Jawarkar, D.T. Mahajan, T.B. Hadda, J. Sheikh, and K.N. Patil, (2011). QSAR and CoMFA studies of biphenyl analogs of the anti-tuberculosis drug (6S)-2-nitro-6-\{[4(trifluoromethoxy)benzyl]oxy\}-6,7-dihydro-5H-imidazo[2,1b][1,3]oxazine(PA-824), Med. Chem. Res., DOI:10.1007/s00044$011-9787-\mathrm{x}$

V.H. Masand, D.T. Mahajan, K.N. Patil, K.D. Chinchkhede, R.D. Jawarkar, T. Ben Hadda, A.M. Alafeefy, and I.G. Shibi, (2012). k$\mathrm{NN}$, quantum mechanical and field similarity based analysis of xanthone derivatives as a-glucosidase inhibitors, Med. Chem. Res, 1-12, DOI:10.1007/s00044-012-9995-Z

K. Papireddy, M. Smilkstein, J.X. Kelly, Shweta, S.M. Salem, M. Alhamadsheh, S.W. Haynes, G.L. Challis, and K.A. Reynolds, (2011). Antimalarial Activity of Natural and Synthetic Prodiginines. J. Med. Chem. 54 : 5296-5306.

N.R. Williamson, P.C. Fineran, F.J. Leeper, and G.P.C. Salmond, (2006). The biosynthesis and regulation of bacterial prodiginines. Nature Reviews 4: 887-899.

P.A. Gale, and W. Dehaen, (2010). Anion Recognition in Supramolecular Chemistry (Topics in Heterocyclic Chemistry), Springer, New York.

M.A. Grishina, A.A. Pogrebnoi, V.A. Potemkin, and T.Y. Zrakova, (2005). Molecular-biological problems of drug design and mechanism of drug action theoretical study of the substrate specificity of cytochrome P-450 isoforms, Pharm. Chem. J. 39(10) 509-513. 
M.A. Grishina, and V.A. Potemkin, (2008). A new paradigm for pattern recognition of drugs, J Comput Aided Mol Des 22 : 489-505.

A.A. Pogrebnoi, M.A. Grishina, V.A. Potemkin, and D.A. Sysakov, (2010). Molecular-biological problems of drug design and mechanism of drug action modeling complexes of substrates with cytochrome P450 2C9, Pharm. Chem. J. 44(5) 237-240.

M.A. Grishina, A.A. Pogrebnoi, V.A. Potemkin, and N.N. Ivshina, (2008). A Study of Conformational States of Substrates of Isoform 3A4 of Cytochrome P-450, Mol. Biophys. 53(5) 758-765.

V.A. Potemkin, R.M. Arslambekov, E.V. Bartashevich, M.A. Grishina, A.V. Belik, S. Perspicace, and S. Guccione, (2002). Multiconformational method for analyzing the biological activity of molecular structures, J. Struct. Chem. 43(6) 1045-1049.

A.A. Pogrebnoi, M.A. Grishina, and V.A. Potemkin, (2009). Modeling of complexes between cytochrome p450 1a2 and substrates, J. Struct. Chem. 50(5) 814-820. 